\title{
Densificação rápida de cerâmicas de $\mathrm{SnO}_{2}$ (Fast densification of $\mathrm{SnO}_{2}$ ceramics)
}

\author{
G. J. Pereira, D. Gouvêa \\ Laboratório de Processos Cerâmicos, Departamento de Engenharia Metalúrgica e de Materiais \\ Escola Politécnica da Universidade de S. Paulo \\ Av. Prof. Mello Moraes, 2463, Cidade Universitária \\ S. Paulo, SP, 05508-900 \\ dgouvea@usp.br
}

Resumo

Os pós à base de óxido de estanho são conhecidos por apresentarem baixa densificação mesmo a temperaturas de sinterização acima de $1500^{\circ} \mathrm{C}$. A introdução de diferentes íons metálicos como $\mathrm{Mn}^{2+}, \mathrm{Fe}^{3+}$ e $\mathrm{Cu}^{2+}$ induzem a redução do volume de poros e crescimento de grãos durante a sinterização. Pós à base de $\mathrm{SnO}_{2}$ foram preparados pela rota química derivada da patente de Pechini, contendo diferentes concentrações de $\mathrm{Mg}^{2+}$ ou $\mathrm{Fe}^{3+}$. Todas as amostras apresentaram uma alta taxa de densificação nos momentos iniciais, quando sinterizadas por "fast firing". As amostras contendo $5 \%$ em mol de ferro sinterizadas durante $30 \mathrm{~s}$ a $1200{ }^{\circ} \mathrm{C}$ apresentaram densidades superiores às amostras sinterizadas por aquecimento em taxas normais $\left(10^{\circ} \mathrm{C} / \mathrm{min}\right)$ por $4 \mathrm{~h}$ a $1200^{\circ} \mathrm{C}$. O fenômeno de eliminação rápida de poros pode ser explicado, levando em conta o papel da superfície no fenômeno de sinterização. A saturação da superfície pelo aditivo foi confirmada por medidas de mobilidade eletroforética dinâmica com base na literatura e em dados experimentais.

Palavras-chave: $\mathrm{SnO}_{2}$, sinterização rápida, aditivos, superfícies.

\section{Abstract}

Tin oxide based powders without additives present low densification even at high sintering temperatures. Different metal cations such as $\mathrm{Fe}^{3+}, \mathrm{Mn}^{2+}$ and $\mathrm{Cu}^{2+}$ are introduced into such powders and induce pore volume reduction during sintering. In this work, $\mathrm{SnO}_{2}$ based powders were prepared with different amounts of $\mathrm{Mg}^{2+}$ and $\mathrm{Fe}^{3+}$ by a polymeric chemical process derived from Pechini's method. All samples presented high initial densification rate when sintered by fast firing. $\mathrm{SnO}_{2}$ samples containing $5 \mathrm{~mol} \%$ of iron ions and sintered by fast firing at $1200^{\circ} \mathrm{C}$ during $30 \mathrm{~s}$ were denser than samples with the same composition but sintered by conventional method, e.g., $1200{ }^{\circ} \mathrm{C} \mathrm{during} 4 \mathrm{~h}$ at $10^{\circ} \mathrm{C} / \mathrm{min}$. The fast densification could be understood considering the role of the surface on the sintering phenomenon. The surface saturation was verified by electrokinetic mobility measurements and reported results.

Keywords: $\mathrm{SnO}_{2}$, sintering, additives, surfaces.

\section{INTRODUÇÃO}

O uso de técnicas de caracterização de superfície tem sido de grande utilidade para o conhecimento das propriedades físicoquímicas dessas interfaces e coloca cada vez mais em evidência a importância destas nas diferentes etapas do processamento cerâmico, tais como beneficiamento, dispersão, conformação e sinterização.

A sinterização de óxido de estanho sem aditivos é caracterizada por uma reduzida densificação mesmo quando as partículas iniciais são muito pequenas (nanométricas) e ainda quando sinterizadas a elevadas temperaturas e longos períodos de tempo [1]. No entanto, uma característica interessante observada no óxido de estanho é a densificação com pequenas quantidades de aditivos [2].

Ao menos para baixas temperaturas, trabalhos recentes têm mostrado que a introdução de aditivos não influencia somente os mecanismos de transporte de massa, mas também as características morfológicas dos pós, como a área de superfície específica e o ponto isoelétrico das suspensões aquosas [3]. O comportamento da superfície foi avaliado em função da alteração dos grupos químicos adsorvidos, e concluiu-se que estes grupos variam com a quantidade e o tipo de aditivo, como resultado da segregação dos aditivos. Aplicando-se um modelo de crescimento de partículas, foi possível mostrar que a superfície dos pós desempenham um papel muito importante na microestrutura dos pós à base de $\mathrm{SnO}_{2}$ contendo íons ferro ou magnésio [3]. A segregação espontânea de aditivos é relacionada com a redução da energia de superfície, pois afeta parâmetros como a área de superfície específica, tamanho de grão e ângulo diedro. No entanto, aditivos segregados não devem alterar, prioritariamente, os mecanismos difusionais. A segregação dos aditivos na superfície do $\mathrm{SnO}_{2}$ já foi também observada para outros íons como manganês, antimônio, nióbio e índio. [2, 4].

A sinterização de $\mathrm{SnO}_{2}$ a altas temperaturas e contendo $\mathrm{MnO}_{2}$ [10], $\mathrm{CuO}$ [5], $\mathrm{Nb}_{2} \mathrm{O}_{5}$ [6], $\mathrm{In}_{2} \mathrm{O}_{3}$ [7], $\mathrm{Fe}_{2} \mathrm{O}_{3}$ [6], $\mathrm{Sb}_{2} \mathrm{O}_{3}$ [8], $\mathrm{Ta}_{2} \mathrm{O}_{5}$ [8], em concentrações que variam de 0,5 a $5 \%$ em mol, já foi estudada. No entanto, o papel exato do aditivo na densificação do $\mathrm{SnO}_{2}$ é raramente estabelecido, exceto para o caso da adição de $\mathrm{CuO}(0,5$ a $1 \%$ em mol) e para $\mathrm{MnO}_{2}(0,3 \% \mathrm{em} \mathrm{mol})$.

No caso de adição de $\mathrm{CuO}$, o papel do aditivo geralmente é atribuído ao aparecimento de uma fase líquida que surge em 
temperaturas ligeiramente inferiores a $1100^{\circ} \mathrm{C}$ ao ar [5].

No caso do $\mathrm{MnO}_{2}$, mostrou-se que os cátions estão principalmente segregados na superfície das partículas (nos pós) e dos grãos (nas cerâmicas), e que sua superfície específica pode ser controlada com o aumento da concentração de aditivos. Além disso, mostrou-se que não há relação entre a quantidade de aditivo utilizada e solubilização na rede cristalina [9].

Uma vez que a solubilidade do manganês na rede do $\mathrm{SnO}_{2}$ é muito baixa e a concentração é constante, a eliminação da superfície dos grãos leva a um enriquecimento da superfície dos grãos com o aditivo. O início da densificação do $\mathrm{SnO}_{2}$ contendo íons $\mathrm{Mn}^{3+}$ ocorre somente quando uma concentração superficial crítica de aditivos é alcançada, independentemente da temperatura e da concentração total de manganês [10]. Esta evidência leva a suposição de que a sinterização é determinada pelas características superficiais do material, e não apenas por processos difusionais. Entretanto, a difusão deve ser suficiente para promover o transporte de massa necessário. Assim, é aceitável a suposição de que a concentração crítica de aditivo na superfície pode ser alcançada quando um pó de elevada área de superfície específica contenha alta concentração de aditivos e que todo aditivo permaneça somente na superfície. Sendo a saturação a condição necessária para que haja densificação, esta deve ocorrer de forma muito rápida, principalmente quando o material é aquecido rapidamente.

Este trabalho propõe demonstrar que os efeitos de segregação de íons $\mathrm{Fe}^{3+}$ ou $\mathrm{Mg}^{2+}$ no $\mathrm{SnO}_{2}$, obtidos pelo método Pechini [11], tem uma influência fundamental não somente na superfície específica do pó sintetizado como também no processo de densificação do material.

\section{PROCEDIMENTO EXPERIMENTAL}

A descrição detalhada da obtenção e caracterização dos pós de $\mathrm{SnO}_{2}$ puro ou contendo magnésio ou ferro foi reportada em trabalho anterior [3]. Os materiais de partida foram pós à base de $\mathrm{SnO}_{2}$ puros e contendo 2, 5 e $10 \%$ em mol de Fe, e 2, 5 e $7 \%$ em mol de $\mathrm{Mg}$, obtidos pelo método derivado da patente de Pechini [11].

$\mathrm{O}$ cálculo do tamanho médio de partículas, $\mathrm{D}_{\mathrm{BET}}$, foi feito a partir das medidas de $\mathrm{S}_{\mathrm{BET}}$ (realizadas no equipamento Gemini III 2375 Surface Area Analyser da Micromeritics), considerado uma aproximação esférica para as mesmas, utilizando-se a equação A.

$$
\mathrm{D}_{\mathrm{BET}}=\frac{6}{\rho \cdot \mathrm{S}_{\mathrm{BET}}}
$$

onde Ué a densidade teórica. Nesta equação, $\mathrm{S}_{\mathrm{BET}}$ e $\mathrm{D}_{\mathrm{BET}}$ são dados em $\mathrm{m}^{2} / \mathrm{g}$ e $\mathrm{Pm}$, respectivamente. A presença de aditivos menos densos que o $\mathrm{SnO}_{2}\left(\mathrm{U}_{\mathrm{SnO}_{2}}=6,95 \mathrm{~g} / \mathrm{cm}^{3}\right)$ pode alterar a densidade final do material obtido. Assim, a densidade teórica foi estimada pela seguinte equação B [9]:

$$
\rho_{\mathrm{TEO}}=\frac{\left(1-\mathrm{X}_{\text {aditivo }}\right) \cdot \mathrm{M}_{\mathrm{SnO} 2}+\left(\mathrm{X}_{\text {aditivo }} / \mathrm{n}\right) \cdot \mathrm{M}_{\text {aditivo }}}{\mathrm{V}_{\mathrm{m}}}
$$

onde $\mathrm{Me} \mathrm{M}_{\text {aditivo }}$ são as massas molares dos respectivos óxidos, $\mathrm{n}$ é o número de mols de aditivo por mol de óxido referente ao cátion aditivo e $\mathrm{V}_{\mathrm{m}}$ é o volume molar, que sempre é considerado como igual ao do $\mathrm{SnO}_{2}$ puro $\left(\mathrm{V}_{\mathrm{m}}=21,67 \mathrm{~cm}^{3} / \mathrm{g}\right)$ [12].

A conformação das pastilhas consistiu em prensagem uniaxial com pressão de $392 \mathrm{MPa}\left(\sim 4\right.$ ton/ $\left./ \mathrm{cm}^{2}\right)$ sem qualquer ligante, porém com um pequeno volume de água aplicado diretamente no pó utilizando-se uma seringa. As dimensões médias dos discos a verde utilizados para os estudos de sinterização foram $6 \mathrm{~mm}$ de diâmetro e $2 \mathrm{~mm}$ de espessura.

O volume das amostras foi determinado por meio de medidas da altura, H, e diâmetro, I, nos discos. Em cada amostra foram realizadas três medidas em diferentes posições a fim de minimizar o erro experimental. $\mathrm{O}$ diâmetro médio e a espessura média foram utilizados para calcular a densidade Udas pastilhas. A densidade é calculada admitindo-se formato cilíndrico das amostras. Considerando-se a precisão das medidas de massa (realizadas em balança analítica) e dimensões (realizadas com micrômetro digital), o erro relativo, ' U pode ser estimado como $\pm 1,0 \%$. Somente as amostras com variação menor que $2 \%$ nas densidades das amostras a verde (densidade média a verde $r 0,05 \mathrm{~g} / \mathrm{cm}^{3}$ ) foram utilizadas para o estudo.

A sinterabilidade das amostras à base de $\mathrm{SnO}_{2}$ contendo aditivos foi avaliada, realizando-se os ciclos de tratamento térmico em um forno tubular que permite o aquecimento rápido da amostra e também se utilizando uma placa de platina como porta-amostra, o que diminui ao máximo a inércia térmica. As amostras foram introduzidas lentamente na região de $500^{\circ} \mathrm{C}$ do forno, objetivando a eliminação de toda a água adsorvida no material. As amostras foram introduzidas da região de $500^{\circ} \mathrm{C}$ para a região de $1200^{\circ} \mathrm{C}$ em menos de cinco segundos, quando a cronometragem foi iniciada. Após o tempo de sinterização, a placa de platina contendo a amostra foi retirada imediatamente do forno e resfriadas rapidamente. Todo o processo foi realizado ao ar, e os resultados expressos em termos da densidade relativa UU $\bigcup_{t}$. Para o procedimento de sinterização convencional, as amostras foram previamente colocadas no forno à temperatura ambiente $\mathrm{e} o$ mesmo programado para realizar um ciclo de queima convencional, ou seja, $10^{\circ} \mathrm{C} / \mathrm{min}$ até $1200{ }^{\circ} \mathrm{C}$, com patamar de $4 \mathrm{~h}$.

\section{RESULTADOS E DISCUSSÃO}

A Tabela I compila os valores das densidades relativas (UU) obtidas para pastilhas de óxido de estanho com e sem aditivos, a verde e sinterizadas durante 4 horas a $1200^{\circ} \mathrm{C}$.

A sinterização do $\mathrm{SnO}_{2}$ apresenta como principal característica a ausência de densificação, o que durante o processo de sinterização corresponderia predominantemente a mecanismos de evaporaçãocondensação ou difusão superficial [13]. Atendendo a esta característica, as amostras de $\mathrm{SnO}_{2}$ puro apresentaram uma retração volumétrica muito pouco significativa. Esta retração pode ser associada com um rearranjo das partículas, estabelecendo contatos interparticulares nas regiões mais energéticas da superfície, como descrito pelos modelos de estágio inicial da sinterização.

Podemos observar que para as amostras de $\mathrm{SnO}_{2}$ contendo aditivos, ocorre densificação após 4 horas de tratamento térmico. As amostras contendo $10 \%$ em mol de Fe ou $7 \%$ de $\mathrm{Mg}$ chegaram a apresentar mais de 95\% da densidade teórica estimada, com leves discrepâncias em relação às amostras com menor concentração de aditivos. 
Tabela I - Densidades de pastilhas à base de $\mathrm{SnO}_{2}$ contendo diferentes concentrações de ferro ou magnésio.

[Table I-Densities of $\mathrm{SnO}_{2}$ based pellets containing different amounts of iron or magnesium.]

\begin{tabular}{ccc}
\hline $\begin{array}{c}\text { Amostra } \\
\mathbf{S n O}_{2}\end{array}$ & $\begin{array}{c}\text { Verde } \\
\left(\mathbf{U U} \mathbf{U}_{\mathbf{t}}\right) \%\end{array}$ & $\begin{array}{c}\text { 4 horas } \\
(\boldsymbol{U U}) \boldsymbol{t})\end{array}$ \\
\hline $0 \%$ de aditivo & 46,1 & 51,7 \\
\hline $2 \% \mathrm{Fe}$ & 47,0 & 91,7 \\
\hline $5 \% \mathrm{Fe}$ & 46,2 & 91,6 \\
\hline $10 \% \mathrm{Fe}$ & 47,5 & 95,6 \\
\hline $2 \% \mathrm{Mg}$ & 45,6 & 93,3 \\
\hline $5 \% \mathrm{Mg}$ & 46,3 & 95,2 \\
\hline $7 \% \mathrm{Mg}$ & 45,0 & 96,4 \\
\hline
\end{tabular}

Os pós à base de $\mathrm{SnO}_{2}$ contendo íons ferro ou magnésio mostraram-se reativos e uma densificação bastante importante foi observada quando comparada ao óxido de estanho puro. Uma vez que os aditivos utilizados confirmaram suas características de aditivos de densificação para o $\mathrm{SnO}_{2}$, passou-se a verificar a influência da saturação da superfície no fenômeno de densificação. Foi mostrado que para as concentrações de 5 e $10 \%$ em mol de aditivos exista um estado próximo à saturação da superfície para os pós calcinados a $500^{\circ} \mathrm{C}[3,12]$. As amostras foram submetidas a ciclos rápidos de tratamentos térmicos e os resultados de cinética de densificação para as amostras contendo ferro ou magnésio como aditivos são mostradas nas Figs. 1 e 2.

A densificação do $\mathrm{SnO}_{2}$ contendo aditivos é um processo que ocorre de forma muito rápida, ou seja, nos primeiros segundos do tratamento térmico. No caso da adição de $10 \%$ em mol de íons Fe, apenas $10 \mathrm{~s}$ são suficientes para a eliminação de quase $45 \%$ do volume de poros, e chegando a $50 \%$ em 30 s de tratamento térmico. Para a adição de 5\% em mol de íons Fe, o processo ocorre em uma taxa um pouco menor nos primeiros segundos; no entanto, em 30 segundos de tratamento térmico, restam aproximadamente $8 \%$ de porosidade, ou seja, equivalente ao tratamento por $4 \mathrm{~h} \mathrm{em}$

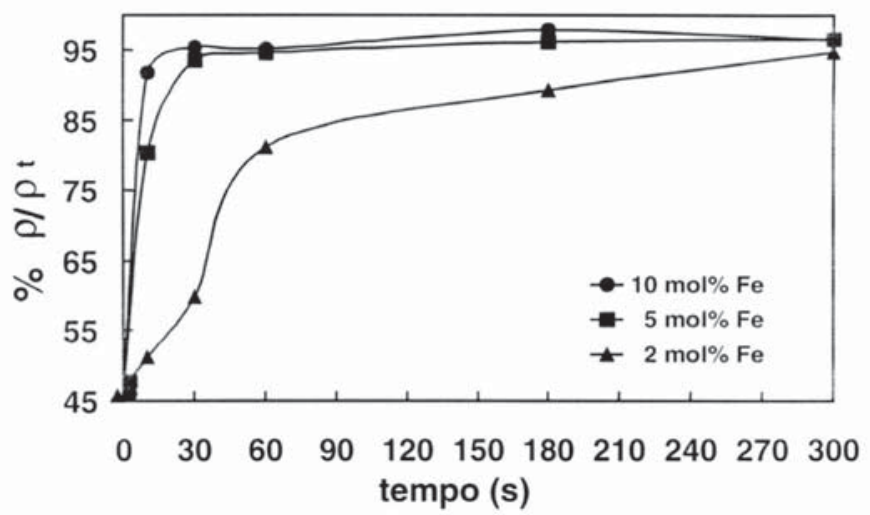

Figura 1: Isotermas de sinterização de cerâmicas à base de $\mathrm{SnO}_{2}$ contendo diferentes concentrações de ferro e sinterizadas a $1200{ }^{\circ} \mathrm{C}$ sob altas taxas de aquecimento e curtos períodos de tempo.

[Figure 1: Sintering Isotherms of $\mathrm{SnO}_{2}$ based ceramics containing different amounts of iron ions. The compacts were sintered at $1200{ }^{\circ} \mathrm{C}$ using high heating rates and short periods of time.] sinterização convencional. Para tempos superiores a $60 \mathrm{~s} \mathrm{a}$ densidade é superior àquela obtida por sinterização convencional.

Um comportamento distinto é observado para o caso da adição de $2 \%$ em mol de íons Fe. A sinterização desta amostra apresenta uma taxa de densificação reduzida nos primeiros $30 \mathrm{~s}$ em relação às amostras com maiores concentrações de aditivo. A partir do primeiro minuto de sinterização, a taxa aumenta significativamente, tendendo a uma continuada eliminação de poros e, após $300 \mathrm{~s}$ de sinterização, uma porosidade semelhante às amostras contendo $5 \mathrm{e}$ $10 \% \mathrm{em}$ mol de $\mathrm{Fe}$. Vale a pena lembrar que nas amostras contendo 5 e $10 \%$ em mol de Fe, a superfície apresenta-se muito próxima da condição de saturação [3, 12]. Contudo, para alcançar a condição de saturação superficial nas amostras contendo $2 \% \mathrm{em} \mathrm{mol} \mathrm{de} \mathrm{Fe,}$ deve haver um crescimento dos grãos. Considerando que todo o aditivo permanece segregado na superfície do pó, a concentração crítica deve ser alcançada após alguns segundos de tratamento térmico, e a partir deste ponto a taxa de densificação passa a ser cada vez maior, levando assim a uma densidade final semelhante às amostras contendo 5 e $10 \%$ de aditivo.

A hipótese da saturação da superfície após o crescimento inicial dos grãos das amostras contendo $2 \% \mathrm{em}$ mol de Fe pode ser confirmada pela medida de mobilidade eletroforética dinâmica pelo método ESA. Como os resultados do sinal ESA são dependentes da composição química da superfície [14], é esperado que esta medida seja sensível às alterações acarretadas pela segregação dos íons na superfície, como observado anteriormente [3]. A Tabela II mostra o deslocamento do ponto isoelétrico dos pós contendo a mesma quantidade de aditivos, porém submetidos a diferentes temperaturas de calcinação, $T_{c}=500,700$ e $900^{\circ} \mathrm{C}$, resultando em pós com diferentes tamanhos de partícula, calculados a partir das medidas de área de superfície específica pelo método BET.

As amostras contendo $2 \%$ em mol de Fe e com tamanho de partícula pequeno, possuem cargas de superfície semelhantes à do dióxido de estanho $\left(\mathrm{pH}_{\mathrm{IEP}}=3,8\right.$ [3]). Isto se deve ao fato de que a superfície não está saturada pelo aditivo. Entretanto, conforme o tamanho de partícula médio aumenta, a concentração de aditivo por unidade de área aumenta, e isto se reflete no valor do ponto isoelétrico, que tende ao do óxido de ferro $\left(\mathrm{pH}_{\mathrm{IFP}}=8,3\right.$ [15]). Este comportamento é similar ao observado para o $\mathrm{SnO}_{2}$ contendo maiores quantidades de ferro [3], demonstrando que a concentração de aditivos na superfície está aumentando.

As isotermas na Fig. 2 mostram a sinterização do $\mathrm{SnO}_{2}$ contendo $2,5 \mathrm{e} 7 \% \mathrm{em}$ mol de $\mathrm{Mg}$ e sinterizadas por aquecimento rápido.

Do mesmo modo que nas amostras contendo Fe como aditivo, a isoterma de sinterização para o sistema $\mathrm{SnO}_{2}+\mathrm{Mg}$ também aponta para uma densificação rápida, porém em uma taxa mais reduzida. Mesmo com $300 \mathrm{~s}$ de tratamento térmico, o sistema tende a uma eliminação gradual da porosidade; no entanto, conforme visto nas medidas feitas para as amostras sinterizadas durante $4 \mathrm{~h}$, a porosidade remanescente deve manter-se em torno de $4 \%$.

Com estes dados, podemos observar que em comparação ao ferro, a adição de magnésio é bastante efetiva na estabilização da superfície a baixas temperaturas [3]. Em contrapartida, a adição do ferro confirma sua efetividade como aditivo de sinterização, promovendo a densificação do óxido de estanho em tempos muito curtos.

Estes resultados sugerem que a sinterização do $\mathrm{SnO}_{2}$ pode ser governada por fenômenos superficiais. Considerando que os 
Tabela II - Tamanho de partícula e ponto isoelétrico de um pó à base de $\mathrm{SnO}_{2}$ contendo $2 \%$ em mol de Fe. Os pós foram calcinados em diferentes temperaturas por $10 \mathrm{~h}$.

[Table II - Particle size and isoelectric point of $\mathrm{SnO}_{2}$ based powders containing $2 \mathrm{~mol} \%$ of $\mathrm{Fe}$. The powders were calcined at different temperatures for $10 \mathrm{~h}$.

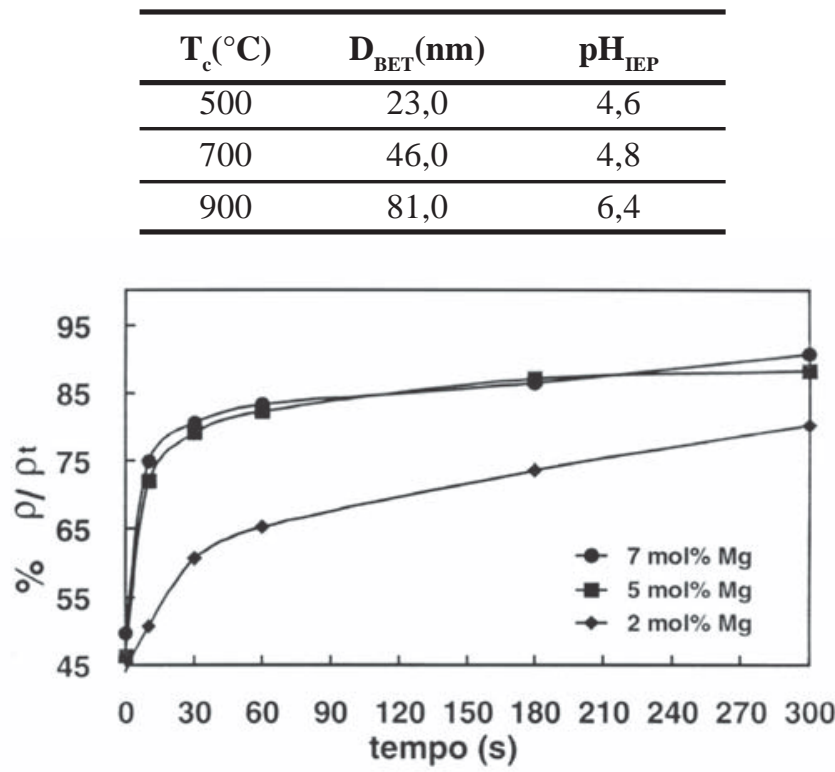

Figura 2: Isotermas de sinterização de pastilhas à base de $\mathrm{SnO}_{2}$ contendo diferentes concentrações de magnésio e sinterizadas a $1200{ }^{\circ} \mathrm{C}$ com altas taxas de aquecimento e curtos períodos de tempo.

[Figure 2: Sintering isotherms of $\mathrm{SnO}_{2}$ based ceramics containing different amounts of magnesium ions. The compacts were sintered at $1200{ }^{\circ} \mathrm{C}$ using high heating rates and short periods of time.]

aditivos ocupam sítios substitucionais na rede, a introdução de cátions com valência menor que 4+ promoveriam um aumento no coeficiente de difusão do sistema, devido à geração de defeitos, responsável pelo equilíbrio do sistema. Uma possibilidade de formação de solução sólida pode ser proposta pelas seguintes reações de formação de defeitos:

$$
\begin{aligned}
& \mathrm{MgO} \longrightarrow \mathrm{SnO}_{2} \longrightarrow \mathrm{Mg}_{\mathrm{Sn}}^{2^{\prime}}+\mathrm{V}_{0}^{2 \cdot}+\mathrm{O}_{0}^{\mathrm{x}} \\
& \mathrm{Fe}_{2} \mathrm{O}_{3} \longrightarrow \mathrm{SnO}_{2} \longrightarrow 2 \mathrm{Fe}_{\mathrm{Sn}}^{\prime}+\mathrm{V}_{0}^{2 \cdot}+3 \mathrm{O}_{0}^{\mathrm{x}}
\end{aligned}
$$

Comparando-se os aditivos em termos das equações de formação de vacâncias, $\mathrm{o} \mathrm{Mg}^{2+}$ formaria o dobro de vacâncias de oxigênio em relação ao $\mathrm{Fe}^{3+}$. Assim, era de se esperar que em um sistema cujo processo de densificação seja controlado unicamente por difusão, a adição de íons magnésio fosse mais efetiva que a adição de íons ferro durante a sinterização. Contudo, o que se observa é exatamente o contrário, sendo necessária, portanto, a consideração do papel da superfície durante a sinterização.

\section{CONCLUSÕES}

Foi possível obter-se pastilhas cerâmicas densas à base de $\mathrm{SnO}_{2}$ contendo $\mathrm{Fe}$ ou $\mathrm{Mg}$ por meio de aquecimento rápido. Apenas $10 \mathrm{~s}$ são suficientes para se alcançar mais de $90 \%$ da densidade teórica utilizando-se ferro como aditivo, enquanto para as amostras contendo magnésio, com $30 \mathrm{~s}$ a densidade relativa alcançada é substancial, mas menor se comparada com as amostras contendo íons ferro. As medidas de área de superfície específica e ponto isoelétrico sugerem que os aditivos estão agindo nas interfaces. Desta forma, o papel da saturação da superfície durante a sinterização do $\mathrm{SnO}_{2}$ deve ser levado em consideração, uma vez que os fenômenos de densificação não podem ser explicados pelos modelos convencionais, que são baseados principalmente em considerações difusionais.

\section{REFERÊNCIAS}

[1] E. R. Leite, J. A. Cerri, E. Longo, J. A. Varela, A. C. Paskocimas, "Sintering of ultrafine undoped $\mathrm{SnO}_{2}$ powder", J. Eur. Cer. Soc. 21 (2001) 669.

[2] D. Gouvea, J. A. Varela, E. Longo, A. Smith, P. Bonnet, "Chemical synthesis of homogeneous $\mathrm{SnO}_{2}$ powders doped with manganese", Eur. J. Solid State Inorg. Chem. 30 (1993) 915.

[3] G. J. Pereira, R. H. R. Castro, P. Hidalgo, D. Gouvea, "Surface segregation of additives on $\mathrm{SnO}_{2}$ based powders and their relationship with macroscopic properties", Appl. Surf. Sci. 195 (2002) 277.

[4] D. Szczuko, J. Werner, S. Oswald, G. Behr, K. Werzig, "XPS investigations of surface segregation of doping elements in $\mathrm{SnO}_{2}$ ", Appl. Surf. Sci. 179 (2001) 301.

[5] J. A. Varela, O. J. Whittemore, E. Longo, "Pore size evolution during sintering of ceramics oxides", Ceram. Int. 16 (1990) 177.

[6] L. Pennisi, "The effect of certain oxides on the sintering and semiconducting nature of $\mathrm{Sb}_{2} \mathrm{O}_{3}$ doped $\mathrm{SnO}_{2}$ ", M.Sc. Diss., Alfred University (1978).

[7] B-C. Kim, J-H. Lee, J-J. Kim, T. Ikegami, "Rapid rate sintering of nanocrystalline indium tin oxide ceramic: particle size effect", Mater. Lett. 52 (2002) 114.

[8] W. C. Las, N. Dolet, P. Dordor, J. P. Bonnet, "Influence of additives on the electrical properties of dense $\mathrm{SnO}_{2}$-based ceramics", J. Appl. Phys. 74 (1993) 6191.

[9] D. Gouvea "Efeito da segregação do manganês na morfologia dos pós e na sinterização do $\mathrm{SnO}_{2}$ ", Tese de Doutorado, Universidade Federal de S. Carlos (1995).

[10] D. Gouvea, A. Smith, J. P. Bonnet, J. A. Varela, "Densification and coarsening of $\mathrm{SnO}_{2}$ based materials containing manganese oxide”, J. Eur. Ceram. Soc. 18, 4 (1998) 345.

[11] M. Pechini, U.S. Pat. n. 3.330.697 (1967).

[12] G. J. Pereira, "Efeitos da segregação dos íons magnésio ou ferro nas características de superfície e na sinterização do $\mathrm{SnO}_{2}$ ", Diss. Mestrado, Universidade de S. Paulo (2002).

[13] W. D. Kingery, M. Berg, "Study of initial stages of sintering solids by viscous flow, evaporation-condensation, and selfdiffusion”, J. Appl. Phys. 26, 10 (1955) 1205.

[14] R. J. Pugh, "Dispersion and stability of ceramic powders/ interparticle interactions in liquids", in Surface and Colloid Chemistry in Advanced Ceramic Processing - Surfactant Science Series, Vol. 51, eds. R. J. Pugh, L. Bergström, Marcel Dekker, New York (1994) 136. [15] C. W. Hoogendam, A. Keizer, M. A. S. Cohen, B. H. Bijsterbosch, "Adsorption mechanism of caboxymethyl cellulose on mineral surfaces", Langmuir 14 (1998) 3285.

(Rec.12/03/03, Ac. 13/06/03) 